\title{
Amphibian species vary in their learned avoidance response to the deadly fungal pathogen Batrachochytrium dendrobatidis
}

\author{
Taegan McMahon ${ }^{1}$, Megan Hill ${ }^{1}$, Garrett Lentz ${ }^{2}$, Electra Scott ${ }^{1}$, Nadia Tenouri ${ }^{2}$, and \\ Jason R. Rohr ${ }^{3}$ \\ ${ }^{1}$ University of Tampa \\ ${ }^{2}$ University of South Florida \\ ${ }^{3}$ Univ S Florida
}

June 2, 2020

\begin{abstract}
Abstract: Lethal and sublethal effects of pathogens should theoretically select for host avoidance of these organisms. Oak toads, for example, learn to avoid the pathogenic fungus Batrachochytrium dendrobatidis (Bd) after one infection-clearance event. Here, we investigated whether four taxonomically distinct amphibians, Cuban treefrogs (Osteopilus septentrionalis), southern toads (Bufo terrestris), greenhouse frogs (Eleutherodactylus planirostris), and pine woods treefrogs (Hyla femoralis) were also able to learn to avoid Bd and, if so, what cues they used to identify Bd. Cuban treefrogs, pine woods treefrogs, and greenhouse frogs did not appear to exhibit detectable innate or learned avoidance of Bd. However, southern toads learned to avoid Bd after only one exposure. Southern toads avoided any treatment containing Bd metabolites but did not avoid treatments that lacked Bd metabolites even when dead zoospores were present. Bd metabolites include digestive enzymes that breakdown host tissue and appear to be the cues that amphibians use to avoid Bd, which is consistent with a Classical or Pavlovian Conditioning response. It appears that not all species respond the same way to $\mathrm{Bd}$, which is important information when developing disease models and conservation plans for amphibians.
\end{abstract}

\section{Amphibian species vary in their learned avoidance response to the deadly fungal pathogen Batrachochytrium dendrobatidis}

Taegan A. McMahon ${ }^{1+}$, Megan N. Hill ${ }^{1 *}$, Garrett C. Lentz ${ }^{2,3^{*}}$, Electra F. Scott ${ }^{1 *}$, Nadia F. Tenouri ${ }^{2,3^{*}}$, Jason R. Rohr ${ }^{2,4}$

\section{Affiliations:}

${ }^{1}$ University of Tampa, Department of Biology, Tampa, Florida 33606, USA.

${ }^{2}$ University of South Florida, Department of Integrative Biology, Tampa, Florida 33620, USA.

${ }^{3}$ University of Otago, School of Geography, Dunedin, 9016, New Zealand 46556, USA.

${ }^{4}$ University of Notre Dame, Notre Dame, Indiana

*Authors contributed equally to this work

Author emails (in order of authorship):tmcmahon@ut.edu, megan.hill8190@gmail.com, garrett.lentz@postgrad.otago.ac.nz, electradoyle93@gmail.com, nadia.tenouri@postgrad.otago.ac.nz, jasonrohr@gmail.com

Running Title: Amphibians can learn to avoid Bd 
Key words: Chytrid fungus, behavioral resistance, amphibian decline, chytridiomycosis, disease ecology, disease avoidance, behavioral avoidance, behavioral ecology, host-parasite interactions, amphibians

Article type: Letters

Word Count (Abstract): 186

Word Count (main text): 4,696

References: 25

Figures: 5

Tables: 1

Conflicts of interest: No conflicts declared

Author statement : all authors agreed to submission of the manuscript and accept the responsibility for the accuracy and integrity of the manuscript.

+ To whom correspondence should be addressed. E-mail: tmcmahon@ut.edu; 813-974-4694

Author contributions: T.A.M. and J.R.R. designed experiments, T.A.M., M.N.H., G.C.L., E.F.S, and N.F.T. conducted experiments, T.A.M and J.R.R. conducted statistical analyses. T.A.M, G.C.L., N.F.T. and J.R.R. wrote the paper, T.A.M, E.F.S., and J.R.R provided funding and all authors provided editorial advice.

Abstract : Lethal and sublethal effects of pathogens should theoretically select for host avoidance of these organisms. Oak toads, for example, learn to avoid the pathogenic fungus Batrachochytrium dendrobatidis (Bd) after one infection-clearance event. Here, we investigated whether four taxonomically distinct amphibians, Cuban treefrogs (Osteopilus septentrionalis), southern toads (Bufo terrestris), greenhouse frogs (Eleutherodactylus planirostris), and pine woods treefrogs (Hyla femoralis) were also able to learn to avoid $\mathrm{Bd}$ and, if so, what cues they used to identify Bd. Cuban treefrogs, pine woods treefrogs, and greenhouse frogs did not appear to exhibit detectable innate or learned avoidance of Bd. However, southern toads learned to avoid Bd after only one exposure. Southern toads avoided any treatment containing Bd metabolites but did not avoid treatments that lacked Bd metabolites even when dead zoospores were present. Bd metabolites include digestive enzymes that breakdown host tissue and appear to be the cues that amphibians use to avoid $\mathrm{Bd}$, which is consistent with a Classical or Pavlovian Conditioning response. It appears that not all species respond the same way to $\mathrm{Bd}$, which is important information when developing disease models and conservation plans for amphibians.

\section{Introduction}

Emerging infectious diseases are one of the largest threats to global biodiversity, but despite this, disease is historically one of the least studied issues in conservation (Lawler et al. 2006). Amphibians, for example, are one of the most threatened vertebrate taxon (Stuartet al. 2004) and have experienced extreme global declines because of the pathogenic fungus Batrachochytrium dendrobatidis $(\mathrm{Bd})$. This pathogen is associated with the extinction and extirpation of hundreds of amphibian species globally and remains an imminent and persistent threat to many extant species (Scheele et al. 2019). Additionally, Bd is unlikely to be extirpated given its nearly global distribution and the presence of non-amphibian hosts (McMahon et al. 2013; Brannelly et al. 2015; Scheele et al. 2019).

$\mathrm{Bd}$ has a mobile zoospore stage that produces proteolytic enzymes that degrade elastin proteins in the epidermal layer of the host (Symondset al. 2008; Moss et al. 2010), as well as polyamine spermidine, methylthioadenosine, and tryptophan, all of which are immunomodulators (Rollins-Smith et al. 2015; Rollins-Smithet al. 2019). When crayfish were exposed to Bd metabolites in the absence of the fungus, the metabolites induced crayfish mortality via gill damage (McMahon et al. 2013). Additionally, tadpoles exposed to Bd metabolites in the absence of infection had increased developmental speed (Romansic et al. 2011; McMahon 
et al.2019); tadpoles can utilize developmental plasticity to avoid an unsuitable environment (Newman 1988; Rohr et al. 2004). Although admittedly anthropomorphic, the damage to host tissue caused by the enzymes in the metabolites is likely painful. This could set the stage for learned avoidance of Bd through Classical or Pavlovian Conditioning. An innate response, in this case avoidance of pain, is paired with a second stimulus, cues from $\mathrm{Bd}$, to induce learned avoidance of $\mathrm{Bd}$. There is precedent for this, as we already know amphibians can learn to avoid pathogenic fungi. For example, bullfrog (Rana catesbeiana) tadpoles use chemical cues to avoid conspecifics infected with the fungus Candida humicola (Kiesecker et al. 1999), and oak toads (Bufo quercicus ) learn to avoid $\mathrm{Bd}$ after experiencing just one $\mathrm{Bd}$ infection and clearance event (McMahon et al. 2014).

While oak toads can learn to avoid Bd after exposure (McMahon et al. 2014), we do not know how common this learned avoidance response is across amphibian species, nor do we know what specific $\mathrm{Bd}$ cues elicit the avoidance response. Here, we tested whether four species of amphibians - Cuban treefrogs (Osteopilus septentrionalis ; family: Hylidae), southern toads (Bufo terrestris ; family: Bufonidae), greenhouse frogs (Eleutherodactylus planirostris ; family: Eleutherodactylidae), and pine woods treefrogs (Hylafemoralis ; family: Hylidae) - exhibited innate or learned avoidance of Bd. We hypothesized that, like oak toads, all four species would learn to avoid $\mathrm{Bd}$ after exposure to and clearance of the pathogen. For any species that exhibited avoidance behavior, we then exposed Bd-naïve and Bd-experienced individuals to different components of $\mathrm{Bd}$ to determine which cues are used to avoid Bd. Generally, we crossed the presence and absence of Bd metabolites with the presence and absence of live and dead zoospores. Given that metabolites are likely painful because they damage amphibian skin, we predicted that the metabolites would be an important component of $\mathrm{Bd}$ that triggered any learned avoidance response. If amphibians learn to avoid $\mathrm{Bd}$ without exposure to live infectious propagules, then it would suggest that a vaccine including a non-infectious cue, such as metabolites, might be effective at inducing an acquired behavioral resistance response that could increase the likelihood that amphibians could persist with Bd.

Materials and Methods

Animal Husbandry

Cuban treefrogs (Osteopilus septentrionalis ; adults), southern toads (Bufo terrestris ; metamorphs), greenhouse frogs (Eleutherodactylus planirostris ; adults), and pine woods treefrogs (Hyla femoralis ; metamorphs), were collected within $10 \mathrm{~km}$ of the University of Tampa (Tampa, FL). The large arboreal species (Cuban treefrogs) were housed individually in 1-L plastic cups and all other species (pine woods treefrogs, greenhouse frogs and southern toads) were housed in smaller plastic containers $(\sim 8 \times 11 \mathrm{~cm})$. All animals were maintained with paper towels moistened with artificial spring water (ASW; Cohen et al.1980) and fed gutloaded crickets dusted with Rep-Cal vitamins ad libitum. Their bedding and containers were changed weekly (new containers were used for each behavioral trial). Animals were maintained at $23^{\circ} \mathrm{C}$ until the start of the experiment and were then maintained at $18^{\circ} \mathrm{C}$ unless otherwise noted.

\section{Treatment Inoculate Preparation}

We cultured two strains of Bd (JEL 423 isolated in Panamá fromHylomantis lemur and SRS 810 isolated in the USA from Rana catesbeiana) on $1 \%$ tryptone agar plates. Each amphibian species tested was exposed to the strain that was isolated closest to their native range (Table 1). Immediately before use in the experiments, the $\mathrm{Bd}+$ plates were flooded with ASW to suspend the infectious zoospores. The ASW from all Bd+ plates was homogenized, zoospore concentration was quantified using a hemocytometer, and the stock was diluted with ASW to $1.5 \times 10^{5}$ zoospores $/ \mathrm{mL}(\mathrm{Bd}+$ stock). Bd-free $1 \%$ tryptone agar plates were flooded using the same technique to create a control (Bd- stock).

We used the same methodology to prepare the different treatment inoculates for all the amphibian species. To create Dead zoospores with metabolites, the $\mathrm{Bd}+$ stock was flash frozen with liquid nitrogen. To create Dead zoospores alone, the Dead zoospores with metabolites stock was filtered through a $1.2 \mu \mathrm{m}$ filter (GE Whatman Laboratory Products, Springhill, PA) to separate the zoospores from the liquid containing the metabolites. We washed the zoospores off the filter with an equivalent amount of ASW. To create Metabolites 
alone, we used the filtrate separated from the Dead zoospores with metabolites stock (see Dead zoospores alone ). For all treatments we used a hemocytometer to verify that we had what we expected, either live zoospores, dead zoospores, or no zoospores. For the dead Bd and metabolite treatments, we verified there was no live fungus by plating $1 \mathrm{~mL}$ of the treatment inoculate on three $1 \%$ tryptone agar plates and monitored for growth for two weeks (there was no Bd growth).

\section{Bd-Infection and Clearance}

Each frog was exposed to their respective treatment (see Treatment Inoculate Preparation) as a Bd-naïve individual and then again as a Bd-experienced individual (except for the control treatment groups) to determine changes in individual behavior. Below we describe the treatments given to each group and then describe how data were collected. To create Bd-experienced hosts, individuals were infected with and cleared of a sublethal dose of live Bd (Fig. 1). Live Bd from the Bd+ stock was pipetted on the dorsal surface of each amphibian within their housing chamber (not the experimental chamber) so the Bd could not be avoided. After exposure, all amphibians (including all uninfected controls) were then held at $18^{\circ} \mathrm{C}$ for $11 \mathrm{~d}$, allowing enough time for growth of the Bd infection but not enough time for Bd to cause morbidity (McMahon et al. 2014). Amphibians were then cleared of infections using the heat clearance method described by McMahon,et al. (2014). Briefly, all amphibians were held at $23^{\circ} \mathrm{C}$ for $3 \mathrm{~d}$ (to reduce temperature shock) and were then moved to a $30^{\circ} \mathrm{C}$ incubator for $11 \mathrm{~d}$ (to clear the infection). Afterward, they were moved to $23^{\circ} \mathrm{C}$ for $3 \mathrm{~d}$. before finally returning to $18^{\circ} \mathrm{C}$ for the remainder of the experiment (see Fig. 1 for experimental timeline).

\section{Behavioral Observations for all Amphibian Species}

In the observational choice chamber, Greenhouse frogs and pine woods treefrogs were exposed to Live $B d$ or Control treatments ( $n=13$ or 9 amphibians/treatment, respectively; Table 1, Fig. 1) as Bd-naïve and Bd-experienced individuals. Southern toads and Cuban treefrogs were exposed to one of five treatments: Live $B d$, Control, Dead zoospores with metabolites, Dead zoospores alone, andMetabolites alone. The amphibians were randomly assigned to one of the treatments $(n=20$ and 15 amphibians/treatment, respectively; Table $1)$.

Observation chambers were created by placing ASW moistened paper towels on each side of the container leaving a $1 \mathrm{~cm}$ gap in the center. For the Cuban treefrogs, the paper towels and gap covered the bottom of the container and extended vertically up the wall so that the treefrogs could not avoid the two treatments. Within each container, the paper towel on one side of the chamber was inoculated with $1 \mathrm{~mL}$ of the treatment inoculate (see Treatment Inoculate Preparation; Table 1) and the paper towel on the other side received $1 \mathrm{~mL}$ of ASW. The side inoculated with the treatment was selected randomly and we implemented a double-blind inoculation process so that the person running the behavioral trials did not know the treatment location. For each behavioral trial, the amphibian was placed in the center of the container. After a 20-minute acclimation period, we recorded the location of the amphibians in the container (left, center, or right) every 15 min for 3-4 h depending on the species (southern toads: $4 \mathrm{hrs}$; all other species: $3 \mathrm{hr}$ ).

\section{Statistical Analysis}

Statistics were analyzed with R statistical software (Development Core Team 2013) and significance was attributed at $P<0.05$. The greenhouse frogs and pine woods treefrogs were analyzed together because they were conducted simultaneously. Behavioral trials were conducted separately for the Cuban treefrogs and southern toad and thus they were analyzed using independent statistical analyses. For the Cuban treefrog and the southern toad, we conducted general linear mixed effects models (package: nlme, function: lme) with individual amphibian as a random effect (to account for the lack of independence of repeated behavioral observations on an individual), proportion of observations avoiding during a trial as the response variable (arcsine square root-transformed, unfortunately we could not get models with a binomial error distribution to consistently converge), mass as a covariate, and treatment and experience status (naïve versus experienced to $\mathrm{Bd}$, nested within individual as random effects) as crossed independent variables. We followed these analyses with targeted hypothesis testing where we pooled all treatments containing $i$ ) any zoospores, ii ) dead zoospores, and iii ) metabolites in an effort to assess whether any avoidance response was induced by the 
actual process of infection, physical contact with Bd zoospores, or Bd metabolites, respectively. There were too few greenhouse frogs and pine woods treefrogs to have frogs assigned to treatments with controls solutions assigned to both sides of the container as we did for the Cuban treefrogs and southern toads. Thus, for the greenhouse frogs and pine woods treefrogs, we tested for deviations between the observed and expected null spatial distributions and tested for differences between these two species in their responses because they were tested simultaneously. Probability values were determined using the likelihood ratio tests based on Type II sums of squares (package: car, function: Anova). Amphibians that died during the experiment were excluded from analyses.

\section{Results}

\section{Cuban Treefrog}

Cuban treefrogs did not avoid any treatments (Treatment: $d f=4, \chi^{2}=6.66, P=0.15$; Fig. $2 \mathrm{~A}$ ), regardless of whether they were Bd naïve or experienced (experienced: $d f=4, \chi^{2}=0.43, P=0.51$; Treatment*experienced: $d f=4, \chi^{2}=5.25, P=0.26$; Fig. 2B). To increase statistical power, we compared treatments with or without: metabolites (Metabolites: $d f=1, \chi^{2}=0.05, P=0.82$; Metabolites*experience: $d f=1, \chi^{2}=4.03, P=0.05$ ), zoospores (Zoospores: $d f=1, \chi^{2}=1.00, P=0.32$; Zoospores*experience: $d f=$ $1, \chi^{2}=0.02, P=0.90$ ), and live Bd (Live Bd: $\chi^{2}=0.62, P=0.43$; Live Bd*experience: $\chi^{2}=1.76, P=0.18$ ). Cuban treefrogs exhibited no evidence of innate or learned avoidance even after pooling these treatments to increase statistical power. Additionally, Cuban treefrogs showed no evidence of avoidance of or attraction to zoospores (dead or alive) or live Bd. Although there was a marginally significant metabolite-by-experience interaction, there was no significant avoidance when naïve or Bd-experienced and experience significantly reduced the strength of the avoidance rather than enhanced it. Thus, this statistical result is not consistent with innate or learned avoidance to Bd.

\section{Greenhouse Frog and Pine woods Treefrog}

Bd-naïve greenhouse frogs and pine woods treefrogs did not avoid the live Bd treatment (Treatment: $\chi^{2}=$ 0.007, $P=0.93$; Figs. 3A, 3B) and there was no difference in behavioral response between the two species when Bd-naïve (Species: $\chi^{2}=1.9, P=0.17$ ). Nine out of 10 pine woods treefrog died during the live Bdexposure and so we could not determine whether exposure to and clearance of $\mathrm{Bd}$ affected their behavioral response. Thus, we could only test for the effect of experience on greenhouse frogs. The Bd-experienced greenhouse frogs did not avoid the live Bd treatment (Treatment: $\chi^{2}=0.55, P=0.46$, Fig. 3B). In fact, they exhibited $4.5 \%$ more attraction to the $\mathrm{Bd}$ after experience, indicating that there was no trend for learned avoidance.

\section{Southern Toad}

Bd-naïve toads did not avoid of any of the treatments (Treatment: $d f=4, \chi^{2}=2.00, P=0.736$; Fig. 2C). There was a significant effect of treatment on the avoidance behavior of Bd-experienced toads (Treatment: $d f=4, \chi^{2}=2.40, P=0.018$, Fig. $2 \mathrm{D}$ ) but they did not avoid all treatments equally. Regardless of whether toads were Bd-naïve or Bd-experienced, toads showed no significant differential avoidance of treatments with or without zoospores (Zoospores: $d f=1, \chi^{2}=0.11, P=0.742$; Zoospores*experience: $d f=1, \chi^{2}$ $=0.12, P=0.729$; Fig. $4 \mathrm{~A}$ ) or with or without live zoospores (Live zoospores: $d f=1, \chi^{2}=0.03, P=$ 0.854; Live zoospores*experience: $d f=1, \chi^{2}=0.33, P=0.568$; Figs. $\left.2 \mathrm{D}, 4 \mathrm{~B}\right)$. However, avoidance of $\mathrm{Bd}$ metabolites was significantly greater when toads were $\mathrm{Bd}$-experienced than Bd-naïve (Metabolite* experience: $d f=1, \chi^{2}=5.89, P=0.015$; Figs. $\left.4 \mathrm{C}, 5\right)$, suggesting that the metabolites that contain enzymes that digest amphibian skin trigger a learned avoidance response. To better estimate the magnitude of learned avoidance of treatments with metabolites, we compared treatments with metabolites to only the controls (rather than all treatments without metabolites). In these analyses, the difference between avoidance when experienced and naïve was even stronger than in the previous analyses (Metabolite*experience: $d f=1, \chi^{2}=7.76, P=$ 0.005). Toad responses to the three treatments containing metabolites did not differ significantly when naïve or experienced (Treatment: $d f=2, \chi^{2}=1.06, P=0.589$; Treatment*experience: $d f=2, \chi^{2}=1.69, P=$ 0.430 ), indicating that the overall learned avoidance of metabolites was not a function of just one of these 
three metabolites-containing treatments.

\section{Discussion}

We found that amphibian species had different abilities to learn to avoid Bd. Neither Bd-naïve nor Bdexperienced Cuban treefrogs, greenhouse frogs and pine woods treefrogs avoided Bd. Bd-naïve southern toads exhibited no avoidance of Bd zoospores or metabolites, but after one Bd infection and clearance, they avoided treatments containing Bd metabolites. Oak toads were also capable of learning to avoid Bd (McMahon et al. 2014) and thus this is the second species in Bufonidae capable of acquired behavioral avoidance of $\mathrm{Bd}$. More research is needed to explore whether the ability to learn avoidance behaviors is influenced by phylogeny.

$\mathrm{Bd}$ metabolites triggered the associational behavioral avoidance in southern toads, which is not surprising given that many vertebrate species are capable learning to avoid a cue that is accompanied by a painful stimulus (Dunlop et al. 2006). The metabolites contain proteolytic enzymes and other chemicals that degrade host tissue (Mosset al. 2010; McMahon et al. 2013; Rollins-Smith et al. 2015; Rollins-Smith et al. 2019), and thus exposure to metabolites might indeed be painful. Amphibians may be able to detect these chemical metabolites through the chemo- or pain receptors in their skin (Hillyard \& Willumsen 2011), which could facilitate the detection and avoidance of external stimuli through epidermal contact (for example see Kiesecker et al. 1999). Pavlovian conditioning predicts that pain inducing chemicals would produce a strong behavioral avoidance response. This is not only consistent with what we found here but is also consistent with amphibian behavioral avoidance of other directly transmitted pathogens (Kiesecker et al. 1999, Zylberberg et al. 2013). In all of these cases, the behavioral response is likely to lower exposure to pain, which may also lower risk of infection.

Interestingly, the other species of amphibians tested did not exhibit avoidance of Bd when Bd naïve or Bd experienced. The high Bd-induced mortality in the pine woods treefrogs was not unusual for species in the family Hylidae (Scheele et al. 2019). Given this high susceptibility to Bd, we would have expected strong selective pressure for innate behavioral avoidance, but none was detected in this species. This is possibly because these populations may not have an evolutionary history with Bd, which would be necessary for selection of avoidance responses.

We did not see high Bd-induced mortality in the greenhouse frogs and Cuban treefrogs. Because these species appear to be somewhat tolerant to Bd, they may not have experienced strong selective pressures to exhibit innate or learned avoidance of the fungus. Selection pressures are a product of variation in both exposure and susceptibility to the fungus (Sears et al. 2015). In addition to different selection pressures for avoidance, there might also be differences among species in their ability to learn. Hence, the combination of variation in selection pressures and learning abilities might explain the observed variation among amphibian species in their learned avoidance responses to Bd. We encourage additional research that screens for the ability to discriminate between innate and learned avoidance of pathogens and that identifies the cues used to induce any avoidance response. This would give researchers a better understanding of the strength and importance of behavioral avoidance in the field.

Given that human-assisted migration is believed to have introduced Bd to new locations around the planet in the last 150 years (for example, Ouellet et al. 2005; Padgett-Flohr \& Hopkins 2009; Tallleyet al. 2015), we suspect that many hosts might not have had an evolutionary history with Bd for avoidance behaviors to have become fixed as innate traits. Consequently, we encourage additional research that discriminates between innate and learned avoidance of pathogens and that identifies the cues used to induce any avoidance response. Moreover, there is a need to better understand the strength and importance of Bd avoidance in the field and the degree of species-level variation in this avoidance.

This research has important implications for the conservation and management of amphibian populations threatened by Bd. The IUCN Amphibian Ark network and other conservation initiatives rescued hundreds of amphibian species threatened by Bd and are maintaining these species in captivity in the hopes that they might be released back into the wild (Venesky et al. 2012; Venesky et al. 2013). Reintroduction attempts 
have often failed presumably because of the persistence of Bd in the environment on tolerant hosts (Venesky et al. 2012; McMahon et al. 2013; Venesky et al. 2013). Previous work has suggested that immunization with dead Bd might induce acquired immunological resistance that could facilitate successful reintroductions of captively bred amphibians (McMahon et al.2014). Given that some amphibians can also learn to avoid $\mathrm{Bd}$ without exposure to live infectious propagules, our work opens the door to using non-infectious cues to induce an acquired behavioral resistance response in captively bred and perhaps even wild amphibians. Acquired behavioral resistance to $\mathrm{Bd}$ could increase the likelihood that certain species can persist in nature with Bd. For this to be successful, a better understanding of which species of amphibians are capable of acquiring immunological or behavioral resistance to $\mathrm{Bd}$ is needed. We hope that induction of acquired immunological and behavioral resistance using non-infectious cues of Bd, like Bd metabolites, can reduce Bdinduced amphibian declines and can facilitate the successful re-establishment of captively bred amphibians into the wild.

\section{Figure Legends}

Fig. 1. Timeline for experimental design.

Fig. 2. Both A) Bd-naïve Cuban treefrogs (Osteopilus septentrionalis ; treatment: $d f=4, \chi^{2}=6.66, P=$ 0.15 ) and B) Bd-experienced Cuban treefrogs did not avoid any treatment (experienced: $d f=4, \chi^{2}=0.43, P$ $=0.51$; treatment*experienced: $d f=4, \chi^{2}=5.25, P=0.26$ ). C) Bd-naïve southern toads (Bufo terrestris ) did not avoid any treatment ( $\left.d f=4, \chi^{2}=2.00, P=0.736\right)$, while $\left.\mathrm{D}\right) \mathrm{Bd}$-experienced southern toads avoided only treatments containing $\mathrm{Bd}$ metabolites (dead zoospores with metabolites, live $\mathrm{Bd}$ and metabolites alone; Metabolite*experience: $\left.d f=1, \chi^{2}=5.89, P=0.015\right)$. Shown are mean avoidance ( \pm SEM) and the dashed line at $70 \%$ and $50 \%$, respectively, represents equal avoidance to treatments. The ${ }^{*}$ designates significant avoidance.

Fig. 3. A) Bd-naïve pine woods treefrogs (Hylafemoralis ) did not avoid live Bd. Due to mortality, there were no Bd-experienced pine woods treefrogs. B) Neither Bd-naïve nor Bd-experienced greenhouse frogs (Eleutherodactylus planirostris ) avoided the live Bd treatment. Shown are mean avoidance $( \pm \mathrm{SEM})$, and the dashed line at $50 \%$ represents equal avoidance to treatments.

Fig. 4. Mean ( \pm SEM) relative avoidance of treatments with or without A) zoospores (live or dead), B) live zoospores, and C) metabolites alone for Bd-naïve or Bd-experienced southern toads (Bufo terrestris). The dashed line at $0 \%$ represents equal avoidance to treatments. Positive values indicate greater avoidance and negative values indicate less avoidance.

Fig. 5. Mean ( \pm SEM) relative avoidance of Bd-Naïve and Bd-experienced southern toads (Bufo terrestris ) exposed to treatments with or without Bd metabolites. The dashed line at $50 \%$ represents equal avoidance to treatments. There was a significant interaction between metabolites*experience (Metabolite*experience: $d f$ $\left.=1, \chi^{2}=5.89, P=0.015\right)$.

\section{Hosted file}

image1.emf available at https://authorea.com/users/329008/articles/456151-amphibian-speciesvary-in-their-learned-avoidance-response-to-the-deadly-fungal-pathogen-batrachochytriumdendrobatidis

Figure 1

Figure 2 .

\section{Hosted file}

image2.emf available at https://authorea.com/users/329008/articles/456151-amphibian-speciesvary-in-their-learned-avoidance-response-to-the-deadly-fungal-pathogen-batrachochytriumdendrobatidis

Figure 3. 


\section{Hosted file}

image3.emf available at https://authorea.com/users/329008/articles/456151-amphibian-speciesvary-in-their-learned-avoidance-response-to-the-deadly-fungal-pathogen-batrachochytriumdendrobatidis

Figure 4.

\section{Hosted file}

image4.emf available at https://authorea.com/users/329008/articles/456151-amphibian-speciesvary-in-their-learned-avoidance-response-to-the-deadly-fungal-pathogen-batrachochytriumdendrobatidis

Figure 5

\section{Hosted file}

image5.emf available at https://authorea.com/users/329008/articles/456151-amphibian-speciesvary-in-their-learned-avoidance-response-to-the-deadly-fungal-pathogen-batrachochytriumdendrobatidis

\section{Hosted file}

image6.emf available at https://authorea.com/users/329008/articles/456151-amphibian-speciesvary-in-their-learned-avoidance-response-to-the-deadly-fungal-pathogen-batrachochytriumdendrobatidis

Acknowledgements : We would like to acknowledge Joyce Longcore for providing the Bd strains. Funds were provided by grants to T.A.M. from the National Science Foundation (IOS-1754862), the National Institutes of Health (1R01GM135935-0, KK 2022) and the University of Tampa (Faculty Development Dana Grant) and to J.R.R. from the National Science Foundation (EF-1241889, DEB-1518681, IOS-1754868) and the National Institutes of Health (R01GM109499, R01TW010286-01).

\section{References}

1.

Brannelly, L.A., McMahon, T.A., Hinton, M., Lenger, D. \& Richards-Zawacki, C.L. (2015). Batrachochytrium dendrobatidis in natural and farmed Louisiana crayfish populations: prevalence and implications. Diseases of Aquatic Organisms , 112, 229-235.

\section{2.}

Cohen, L.M., Neimark, H.L. \& Everland, L.K. (1980). Schistosoma mansoni : Response to cercariae to a thermal gradient. Journal of Parasitology , 66, 362-364.

3.

Development Core Team, R. (2013). R: A language and environment for statiscical computing $R$ Foundation for Statistical Computing, Version 3.1.3.

4.

Dunlop, R., Millsopp, S. \& Laming, P. (2006). Avoidance learning in goldfish (Carassius auratus ) and trout (Oncorhynchus mykiss ) and implications for pain perception. Applied Animal Behavior , 97, $255-271$.

5.

Hillyard, S.D. \& Willumsen, N.J. (2011). Chemosensory function of amphibian skin: integrating epithelial transport, capillary blood flow and behaviour. Acta Physiologica , 202, 533-548. 
6.

Kiesecker, J., Skelly, D.K., Beard, K.H. \& Preisser, E. (1999). Behavioral reduction of infection risk. Proceedings of the National Academy of Science, 96, 9165-9168.

7.

Lawler, J.J., Aukema, J.E., Grant, J.B., Halpern, B.S., Kareiva, P., Nelson, C.R. et al. (2006). Conservation science: a 20-year report card. Frontiers in Ecology and the Environment , 4, 473-480.

8.

McMahon, T.A., Brannelly, L.A., Chatfield, M.W.H., Johnson, P.T.J., Joseph, M.B., McKenzie, V.J. et al. (2013). The chytrid fungus, Batrachochytrium dendrobatidis, has non-amphibian hosts and releases chemicals that cause pathology in the absence of infection.Proceedings of the National Academy of Science , 110, 210-215.

9.

McMahon, T.A., Laggan, N.A. \& HIll, M.N. (2019). Metabolites produced by Batrachochytrium dendrobatidis alter development in tadpoles, but not growth or mortality. Diseases of Aquatic Organisms .

10.

McMahon, T.A., Sears, B.F., Venesky, M.D., Bessler, S., Brown, J., Deutsch, K. et al. (2014). Amphibians acquire resistance to live and dead fungus overcoming fungal immunosuppression. Nature , 511, 224-227.

11.

Moss, A.S., Carty, N. \& Francisco, M.J.S. (2010). Identification and partial characterization of an elastolytic protease in the amphibian pathogen Batrachochytrium dendrobatidis . Diseases of Aquatic Organisms , 92, 149-158.

12.

Newman, R.A. (1988). Adaptive plasticity in development ofScaphiopus couchii tadpoles in desert ponds. Evolution , 42, 774-783.

13.

Ouellet, M., Mikaelian, I., Pauli, B.D., Rodrigue, J. \& Green, D.M. (2005). Historical evidence of widespread chytrid infectionin North American amphibian populations. Conservation Biology, 19, 1431-1440.

14.

Padgett-Flohr, G.E. \& Hopkins, R.L. (2009). Batrachochytrium dendrobatidis, a novel pathogen approaching endemism in central California. Diseases of Aquatic Organisms , 83, 1-9.

15.

Rohr, J.R., Elskus, A.A., Shepherd, B.S., Crowley, P.H., McCarthy, T.M., Niedzwiecki, J.H. et al. (2004). Multiple stressors and salamanders: Effects of an herbicide, food limitation, and hydroperiod.Ecological Applications , 14, 1028-1040.

16.

Rollins-Smith, L.A., Fites, S., Reinert, L.K., Shiakolas, A.R., Umile, T.P. \& Minbiole, K.P.C. (2015). Immunomodulatory metabolites released by the frog-killing fungus Batrachochytrium dendrobatidis. Infeciton and Immunity, 83.

17. 
Rollins-Smith, L.A., Ruzzini, A.C., Fites, J.S., Reinert, L.K., Hall, E.M., Joosse, B.A. et al. (2019). Metabolites involved in immune evasion by Batrachochytrium dendrobatidis include the polyamine spermidine. Infection and Immunity, IAI.00035-00019.

18.

Romansic, J.M., Johnson, P.T.J., Searle, C.L., Johnson, J.E., Tunstall, T.S., Han, B.A. et al. (2011). Individual and combined effects of multiple pathogens on Pacific treefrogs. Oecologia , 166, 1029-1041.

19.

Scheele, B.C., Pasmans, F., Skerratt, L.F., Berger, L., Martel, A., Beukema, W. et al. (2019). Amphibian fungal panzootic causes catastrophic and ongoing loss of biodiversity. Science, 363, 1459-1463.

20.

Sears, B.F., Snyder, P.W. \& Rohr, J.R. (2015). Host life history and host-parasite syntopy predict behavioural resistance and tolerance of parasites. Journal of Animal Ecology , 84, 625-636.

21.

Stuart, S.N., Chanson, J.S., Cox, N.A., Young, B.E., Rodrigues, A.S.L., Fischman, D.L. et al. (2004). Status and trends of amphibian declines and extinctions worldwide. Science, 306, 1783-1786.

22.

Symonds, E.P., Trott, D.J., Bird, P.S. \& Mills, P. (2008). Growth characteristics and enzyme activity in Batrachochytrium dendrobatidis isolates. Mycopathologia , 166, 143-147.

23.

Tallley, B.L., Muletz, C.R., Vredenburg, V.T., Fleischer, R.C. \& Lips, K.R. (2015). A century of Batrachochytrium dendrobatidis in Illinois amphibians (1888-1889). Biological Conservation, 182, 254-261.

24.

Venesky, M.D., Mendelson, J.R., Sears, B.F., Stiling, P. \& Rohr, J.R. (2012). Selecting for tolerance against pathogens and herbivores to enhance success of reintroduction and translocation. Conservation Biology, 26, 586-592.

25.

Venesky, M.D., Raffel, T.R., McMahon, T.A. \& Rohr, J.R. (2013). Confronting inconsistencies in the amphibian-chytridiomycosis system: implications for disease management. Biological Reviews , 89, 477-483. 